\title{
Photophysics of closed- and open-ring isomers of a diarylethene with a carboxylic anchor group
}

S. Kuehn ${ }^{a^{*}}$, S. Friede ${ }^{\text {a }}$, M. Zastrow ${ }^{\text {b }}$, C. Gahl ${ }^{\text {a }}$, K. Rueck-Braun ${ }^{\text {b }}$, T. Elsaesser ${ }^{\text {a }}$

${ }^{a}$ Max-Born-Institut für Nichtlineare Optik und Kurzzeitspektroskopie, Max Born Str. 2A, Berlin D-12489, Germany

${ }^{\mathrm{b}}$ Institut für Chemie, Technische Universität Berlin, Str. d. 17. Juni 135, D-10623 Berlin, Germany

* Corresponding author. Fax: +49 306392 1489. E-mail: skuehn@mbi-berlin.de

We study the transient photophysical properties of a diarylethene with a carboxylic anchor group by a combination of steady-state and ultrafast emission and absorption spectroscopy. After excitation of the closed-ring form, fluorescence with a quantum yield of $10^{-5}$ is observed and decomposed into two kinetic components that originate from different excited states. The $\mathrm{S}_{1}$ state of the closed-ring form shows a lifetime of $1.3 \mathrm{ps}$ and decays mainly by internal conversion to the $\mathrm{S}_{0}$ state of this isomer. This vibrationally hot ground state cools on a time scale of $10 \mathrm{ps}$. 


\section{Introduction}

Diarylethenes form a group of photochromic compounds which build upon the photoinduced pericyclic reaction between cyclohexadiene (CHD) and hexatriene (HT) corresponding to the closed- (c-) and open-ring (o-) isomers of the molecule. Both isomers are stable in their ground state at room temperature as a result of a high energy barrier following from Woodward-Hoffmann rules $[1,2]$. The interconversion typically proceeds via an excited state on the picosecond time scale. The photochemical properties of the photochrome can be modified through a range of substitutions influencing the absorption spectra, fluorescence properties, reaction quantum yields, and the fatigue resistance. Functional dyads can be created by linking these molecules to photochromes and surfaces. This makes the diarylethenes highly attractive for applications such as all-optical information processing and storage.

Such applications require an understanding of the photophysics and the switching processes of the individual components. Here, we focus on an asymmetric diarylethene derivative (1-(2,5-dimethyl-3-thienyl)-2-[2-carboxyl-5-(4methoxyphenyl)-3-thienyl]perfluorocyclopentene, DTE) featuring a methoxyphenyl substituent and a carboxylic anchoring group attached to the thiophene rings of the core switching unit as displayed in Scheme 1. The anchoring group allows for the chemisorption of DTE to a variety of semiconductor oxide surfaces such as silicon dioxide or zinc oxide, rendering the molecule relevant for molecular photonics. The substitution pattern can profoundly affect the fait of a photoexcited photochrome, requiring an analysis of its photochemical and photophysical properties.

Studies of transient emission and absorption properties by time-resolved spectroscopy provide access to the dynamics of photophysical processes and allow for 
a separation of events occurring on different time scales [3, 4]. To date, combined absorption and emission measurements have been restricted to $\mathrm{CHD} / \mathrm{HT}$ based photochromes such as fulgimides [4] while reports on time-resolved fluorescence of diarylethenes are rare. The reasons are twofold. The strong fluorescence from o-form isomers frequently originates from long-lived non-reactive conformers [5] and tends to obscure other emission channels. Also, the high reaction turnover facilitates the analysis of transient absorption data. In some cases, fluorescence related to the ringclosing dynamics has nevertheless been investigated to resolve ambiguities in transient absorption data [6].

The situation is much less clear-cut for the ring-opening reaction. A reaction yield below $1 \%$ in most diarylethenes makes the direct observation of the reaction path in transient absorption by educt depletion difficult and the assignment of time constants to specific processes uncertain. Specifically, long-lived states with low populations are hard to track by transient absorption and their role in the photoreaction is still under debate $[6,7]$. Moreover, the c-form of most diarylethenes has an extremely small fluorescence quantum yield and is generally considered nonfluorescent [8]. However, fluorescence should be detectable at least on the time scale of intramolecular reorganization, as is the case for model systems such as, e.g., malachite green with a fluorescence quantum yield below $10^{-4}$ [9]. As for diarylethenes, Shim et al. [3] have reported combined transient absorption and emission measurements on a bis-methylbenzothiophen (BT) derivative, confirming excited state wave packet motion of the c-from. A diyne bridged dimer of an asymmetric BT derivative studied by Yagi and Irie [8] showed appreciable fluorescence only when one of the switches was in the closed ring form. Other BT derivatives exhibit c-form fluorescence when embedded in a solid matrix [10], 
indicating the relevance of structural relaxation in the emission process. Since these experiments were carried out under steady state conditions it is unclear if the emission is related to the photoreaction. To our knowledge, there are no reports on fluorescence from the fundamental transition of closed form diarylethenes other than BT.

In this Letter, we report time resolved fluorescence properties from a class of diarylethenes which have previously been regarded as non-fluorescent in the c-form. Taking advantage of the high detection efficiency of the method, we identify several distinct excited states of the c-form of DTE and distinguish two emission channels located in the visible red and in the near-infrared spectral range. The former is longlived and has no pendant in absorption transients measured in parallel by femtosecond pump-probe spectroscopy. The near-infrared emission from the $\mathrm{S}_{1}$ state displays a 2 ps decay time which also dominates the transient $\mathrm{S}_{0}-\mathrm{S}_{1}$ absorption of the c-isomer. A slower 10 ps component of the absorption change is related to cooling dynamics in the $\mathrm{S}_{0}$ state. Our findings indicate that intrinsic fluorescence emission of diarylethenes may truly be a more common phenomenon and has been overlooked in the past.

\section{Experimental}

Sample preparation: The DTE was prepared starting from 1-(5-Chloro-2methylthienyl-3-yl)-2-[5-(4-methoxyphenyl)-2-methylthienyl-3-yl]-3,3,4,4,5,5hexafluorocyclopentene [11] according to a published carboxylation reaction procedure [12] in 65\% yield. Analytical data of DTE are given in the Supporting Information. DTE was dissolved in degassed chloroform (Sigma-Aldrich, Product Number 154733) under a nitrogen atmosphere (oxygen content $<3 \%$ ). The concentration was between $2.4 \times 10^{-6}$ and $8 \times 10^{-4} \mathrm{M}$ in the different measurements. Samples containing the open-form isomer (o-DTE) were generated by irradiating the solution with a red power LED source emitting at $625 \mathrm{~nm}$. The absorption spectrum 
was then inspected (Perkin-Elmer Lambda 2S) in the VIS region in order to preclude the presence of any inactive byproduct. Samples containing the c-form isomer (cDTE) were produced by irradiating the solution with a $\mathrm{HeCd}$ laser at $325 \mathrm{~nm}$. The ratios of the isomers in the photostationary state (PSS) at $300 \mathrm{~nm}$ were examined by RP-HPLC and a o/c ratio of 1:99 was determined in the PSS. Fluorescence excitation spectra were recorded on a Varian Cary Eclipse. All time resolved measurements were performed using a $500 \mu \mathrm{m}$ thick flow cell with a micro-gear circulation pump (HMS Micro Systems, MZR 2921) set to a rate of $0.6 \mathrm{ml} / \mathrm{s}$. The sample reservoir (1 $\mathrm{ml}$ volume) was irradiated during the measurement either with a $310 \mathrm{~nm}$ LED (UVTOP-305-BL, $0.2 \mathrm{~mW}$ ) to regenerate c-DTE or with a $625 \mathrm{~nm}$ LED to regenerate o-DTE.

Time resolved fluorescence (TF) measurements: Femtosecond excitation pulses were generated by frequency doubling the output of an optical parametric oscillator (Spectra Physics, Opal) synchronously pumped by a mode-locked Ti:sapphire oscillator (Spectra Physics, Tsunami). Frequency-doubling in a $4 \mathrm{~mm}$ thick beta barium borate $(\mathrm{BBO})$ crystal provided $\sim 100 \mathrm{fs}$ pulses at $628 \mathrm{~nm}$ with a repetition rate of $80 \mathrm{MHz}$. The excitation of the sample was arranged in front-face geometry with a focal spot size of $300 \mu \mathrm{m}$ in the sample. The fluorescence emission was directed to the entrance slit of a spectrometer (Princeton Instruments, Acton SP2300) using a mirror imaging system. A streak camera (Hamamatsu C5680, equipped with an S1 cathode) was used for time resolved detection with a resolution down to 2 ps. Time resolved absorption (TA) measurements: Transient absorption changes were measured in a spectrally degenerate pump-probe scheme arranged in non-collinear geometry with cross-polarized electric field orientations. A Ti:sapphire regenerative amplifier (Coherent RegA 9050) running at a repetition rate of $300 \mathrm{kHz}$ with a pulse 
energy of $3.7 \mu \mathrm{J}$ and a pulse duration of $\sim 40$ fs was used to pump an optical parametric amplifier (Coherent OPA 9850). After frequency-doubling of the signal wave with a $100 \mu \mathrm{m}$ BBO crystal and recompressing, the system provided pulses of $\leq$ $50 \mathrm{fs}$ duration at a center wavelength of $590 \mathrm{~nm}$ with pulse energies of $40 \mathrm{~nJ}$. Beam fractions of $90 \%$ (pump) and $10 \%$ (probe) were focused into the sample with a $10 \mathrm{~cm}$ concave mirror to a focal spot size of approximately $100 \mu \mathrm{m}$. The probe pulse was detected by a Si photodiode and a low noise transimpedance amplifier (StanfordResearch SR570) after passing through a polarizer to reject any residual pump light. The pump beam was chopped at $500 \mathrm{~Hz}$ to improve the sensitivity by lock-in detection (Stanford-Research SR830). The temporal resolution of the TA setup estimated from the rise time of the time-integrating absorption decrease of rhodamine $700\left(8 \times 10^{-5} \mathrm{M}\right.$ in ethanol) was approximately $50 \mathrm{fs}$.

\section{Results}

Steady state absorption spectra of the o- and c-forms of DTE in chloroform are shown in Figure 1a. The lowest o-form absorption band peaks around $290 \mathrm{~nm}$ and extends with a shoulder down to $400 \mathrm{~nm}$. Upon excitation at $305 \mathrm{~nm}$, a quantum yield of the ring closure reaction of $\eta_{\mathrm{o} \rightarrow \mathrm{c}}=0.73 \pm 0.3$ was measured. This figure does not account for the presence of the non-reactive parallel conformer [13] in the initial open state since its thermal equilibrium fraction is not known. The c-form thus produced has a broad fundamental band centered at $600 \mathrm{~nm}$ and higher-lying bands around $380 \mathrm{~nm}$. The c-form reverts to the o-form upon irradiation with visible light. A very low quantum yield of the ring opening reaction of $\eta_{c \rightarrow 0}=(2.8 \pm 0.1) \times 10^{-3}$ was determined for excitation at $632 \mathrm{~nm}$. A monotonous transition of the absorption spectra at different stages of partial conversion (thin lines) implies that the photoreaction 
proceeds without distinct stable intermediate species and negligible formation of byproducts. A clear isosbestic point is located at $325 \mathrm{~nm}$.

Upon illumination with UV light, DTE solutions of both forms emit a readily visible white glow. Spectral analysis shows a featureless fluorescence spectrum peaked at $490 \mathrm{~nm}$ (Figure 1b). Within the experimental accuracy, the emission bands from the o- and the c-form are identical. The fluorescence quantum yield is below $1 \%$ for excitation at $380 \mathrm{~nm}$. Note that the emission of the c-form is located energetically above the fundamental absorption band, in contrast to the Kasha rule. The timeresolved decay of the fluorescence spectrally integrated from 465 to $515 \mathrm{~nm}$ displays two kinetic components with decay times around 70 and 700 ps which are identical for the o- and c-form emission. The fluorescence excitation spectra (Figure 1b) reveal distinct transitions and follow roughly the absorption spectra of the corresponding DTE isomers in both shape and intensity. The o-form shows an enhanced excitation peak around $290 \mathrm{~nm}$ where the emission is roughly twice as strong as for the c-form. Clearly, there is no mirror symmetry between excitation and emission indicating an anomalous Stokes shift.

Excitation of the c-form in the range of its fundamental band results in a weak red-shifted fluorescence emission which has been unknown so far. Displayed in Figure $2 \mathrm{a}$ is the steady state fluorescence spectrum observed after excitation at 632 $\mathrm{nm}$. Excitation of the o-form at the same wavelength leads to a residual emission the origin of which is unclear. Upon photoconversion of the o- to the c-form, however, an articulated increase and red-shift of the fluorescence signal is observed. To estimate the fluorescence quantum yield of the c-form, comparative measurements were performed with the dye malachite green in ethanol showing a quantum yield of $\sim 10^{-4}$ [14]. For the c-form of DTE, we derive a fluorescence quantum yield $\eta_{\mathrm{cDTE}} \sim 10^{-5}$. 
Time resolved fluorescence spectroscopy shows the presence of at least two distinct emission channels. To obtain the pure emission of the c-form, the residual fluorescence of the o-form was recorded under identical conditions and subtracted. The resulting time resolved spectra are shown in Figure $2 \mathrm{~b}$. In the first $10 \mathrm{ps}$ after excitation at $628 \mathrm{~nm}$, there appears a prompt emission extending from the visible red far into the near infrared spectral region. The emission transient integrated from 800 to $950 \mathrm{~nm}$ is displayed in Figure 4b. It is nearly limited by the instrument resolution of 2 ps. Deconvolution with the instrument response function (IRF, dashed line) leads to a dominant component with an estimated decay time of $\tau_{\mathrm{f}}=2 \pm 1 \mathrm{ps}$. A weak slow component on the order of 36 ps most probably arises by crosstalk from the emission centered at shorter wavelengths. For delays longer than $10 \mathrm{ps}$, the emission assumes the stationary spectrum without any further spectral dynamics. The corresponding transient, shown in Figure 3, was obtained by spectral integration over 660-690 nm. It exhibits a triphasic decay with time constants on the order of 31,112 and 3300 ps.

Transient absorption changes at the center of the fundamental band of the cform were measured in femtosecond pump-probe experiments with pulses centered at $590 \mathrm{~nm}$. The data in Figure 4a show an instantaneous bleaching and a subsequent absorption recovery which follows a double-exponential decay with time-constants of $\tau_{1}=(1.3 \pm 0.1)$ ps $(84 \%)$ and $\tau_{2}=(12 \pm 1)$ ps $(16 \%)$. After 10 ps, roughly $80 \%$ of the absorption strength has recovered. A permanent bleach due to the photoreaction from the $\mathrm{c}$ - to the $\mathrm{o}$-form is negligible because of the low reaction yield of $2.8 \times 10^{-3}$.

\section{Discussion}

The carboxyl/methoxyphenyl substituents of DTE lead to a considerable red-shift of the c-form fundamental band in comparison with methyl/phenyl [15], 
methoxyphenyl/phenyl [16] or bis-phenyl [17] derivatives. This red-shift is comparable to that found for the bis-aldehyde [18] derivative and is associated with an extension of the effective conjugation length leading to an electronic stabilization of the c-form [19]. This conjecture is also supported by the rather low yield for the ring-opening reaction. Ern et al. [18] assigned the level structure of their bis-aldehyde derivative by comparison with theoretical calculations. By analogy, we assign the absorption bands of DTE as follows: $\mathrm{S}_{0}-\mathrm{S}_{1} \sim 600 \mathrm{~nm}, \mathrm{~S}_{0}-\mathrm{S}_{2} \sim 400 \mathrm{~nm}$ and $\mathrm{S}_{0}-\mathrm{S}_{3} \sim 380$ $\mathrm{nm}$ for the c-form, and $\mathrm{S}_{0}-\mathrm{S}_{1} \sim 300 \mathrm{~nm}$ and $\mathrm{S}_{0}-\mathrm{S}_{2} \sim 280 \mathrm{~nm}$ for the o-form.

Stokes-shifted violet to green fluorescence from the $\mathrm{S}_{1}$ level of the o-form is not uncommon in diarylethenes. It is typically attributed to a conformer where the thiophene rings are aligned with their methyl groups in parallel [13, 20, 21]. For this conformer, the fast deactivation via ring-closure does not compete with the radiative decay. As a result, the fluorescence lifetime is much longer than the photoreaction time, as found here. However, the blue emission of the c-form of DTE cannot be explained on these grounds since stable non-reactive conformers have not been reported for the c-form isomer.

For the c-form isomer, the emission is located energetically above the $\mathrm{S}_{0-1}$ transition, in violation of the Kasha rule. In fact, the emitting state seems to be completely decoupled from the $\mathrm{S}_{1}$ state of the c-form. Even weak coupling to lower lying states of the c-form would lead to a reduction of the lifetime, which is not observed. A violation of the Kasha rule has been reported by Brust et al. [22] for fulgimides in connection with an energy barrier on the reaction path. In the present case, this behavior points to the existence of a relatively long-lived state common to both isomers and populated via ultrafast internal conversion from higher-lying electronic states. A study of unsubstituted, isolated diarylethene molecules has shown 
that intramolecular relaxation and redistribution of energy can occur on the ultrafast time scale [20]. Judging by the pronounced excitation peak at $380 \mathrm{~nm}$ and the fluorescence spectrum, the emission seems to originate from the methoxy-substituted thiophene moiety [23]. Isomers of methyl and phenly derivatives studied by $\mathrm{Pu}$ et al. $[15,16]$, though blue shifted in energy, show a similar emission behaviour.

The very weak red emission of the c-from (Figure 2a) is observed here for the first time. The time-resolved measurements presented in Figures 2b, 3, and $4 \mathrm{~b}$ reveal a very short-lived component with a decay time between 1 and 2 ps (Figure 4b), and contributions with longer decay times (Figure 3). C-form fluorescence has been observed in chemically linked BT diarylethene dimers $[8,24]$. However, dimerization of DTE can be ruled out at the low concentrations used in our experiments. Moreover, the emission characteristics of c-form DTE are similar to those reported for structurally related diarylethenes without a carboxylic linker $[15,25]$, ruling out a significant influence of the linker groups on the emission behavior. We, thus, assign the long-lived red emission to an electronic state in the c-DTE monomer with a chromophore represented by the core unit of DTE. The lifetime up into the nanosecond range without any spectral dynamics points to a relaxed metastable state just below the $\mathrm{S}_{1}$ level of the c-form which we designate M. Multiple potential wells in the excited state and intermediates have in fact been invoked to explain trends in the ring-closing yield of diarylethenes [7, 26].

The short-lived red emission with a decay time of $1-2 \mathrm{ps}$ is assigned to the $\mathrm{S}_{1}$ state of the c-form. This conclusion is strongly supported by the results of the pumpprobe experiments (Figure 4a). After excitation on the $S_{0}-S_{1}$ transition, we observe a transmission increase, i.e., a decrease of the $\mathrm{S}_{0}-\mathrm{S}_{1}$ absorption which is caused by the depletion of the $\mathrm{S}_{0}$ state and stimulated emission from the $\mathrm{S}_{1}$ state. The initial decay of 
this signal reflects a repopulation of the $\mathrm{S}_{0}$ state with a time constant of 1.3 ps which agrees within the experimental accuracy with the fluorescence decay time. This fact shows that the $\mathrm{S}_{1}$ state decays mainly by internal conversion back into the electronic ground state. If being generated at all, the formation yield of other product species is well below the detection margin. The fast internal conversion rate suggests that a conical intersection (CI) is involved in the relaxation to the ground state as predicted by theory [27]. Consequently, the fluorescence emission occurs while the system moves on the $S_{1}$ potential energy surface from the initially prepared Frank-Condon $\mathrm{F}$ states towards the CI. The large spectral width is in agreement with such a process. The ultrafast time evolution points to an essentially barrierless motion on the excited state potential. After passing through the CI, the molecule ends up in a hot, nonthermal ground state of c-DTE, followed by vibrational cooling on the 10 ps timescale $[28]$.

Incidentally, a similar set of time constants of 2 ps and 12 ps has been observed for the ring-opening dynamics in two other diarylethenes with benzoylphenyl-ethenyl [27] and carboxyl substituents [18]. The 12 ps time constant has been ascribed to a metastable state $\mathrm{P}$ on the $\mathrm{S}_{1}$ potential energy surface which is fed at a 2 ps time constant from the initially excited Franck-Condon states. As a result, the repopulation of the ground state as measured in a pump-probe experiment is dominated by the 12 ps decay constant of the state P.

From transient absorption measurements alone it is unclear if the $12 \mathrm{ps}$ component truly arises from a metastable excited state $\mathrm{P}$ or if it is due to dynamics in the ground state, e.g., a vibrationally excited ground state population produced on a shorter time scale. Time resolved fluorescence spectroscopy offers a means to distinguish these scenarios experimentally. Assuming the existence of an excited state 
$\mathrm{P}$ not too different from $\mathrm{F}$ in its photophysical properties, the 10 -fold longer lifetime would imply a 10-fold increase in the fluorescence quantum yield. Taking the signal strength of the short-lived emission as a benchmark, fluorescence from such a state should be well within the detection limit of the experiment but is not observed here. Another possibility could be a large conformational change in the molecule leading to a dynamic reduction of the actual transition dipole moment on a time scale of $<2 \mathrm{ps}$. It seems plausible, that such a stabilized state, once formed, would survive longer than 12 ps. In our data, we only see luminescence from the state $M$ on the time scale beyond 30 ps. This makes the existence of a state $\mathrm{P}$ with a lifetime of 12 ps unlikely and the assignment of this time constant to vibrational cooling in the $\mathrm{S}_{0}$ state most plausible.

The simplest model to describe the present set of data involves a 3-level scheme depicted in the inset of Figure 4. In this model, the c-form of DTE is photoexcited from the ground state $\mathrm{G}$ into a Franck-Condon state F. From $\mathrm{F}$ the photoreaction to the o-form may proceed with the rate $\mathrm{k}_{\mathrm{FO}}$, the metastable state $\mathrm{M}$ may be populated with the rate $\mathrm{k}_{\mathrm{FM}}$ or the molecule may return directly to the ground state via inner conversion $\mathrm{k}_{\mathrm{FNR}}$ and radiatively by near-infrared fluorescence $\mathrm{k}_{\mathrm{FR}}$. The by far dominating decay channel is the internal conversion back into the electronic ground state, as there is no decay component with a time constant $>30 \mathrm{ps}$ or a permanent bleach observed in the transient absorption signal that would correspond to the fluorescence decay time of M. From the noise level we estimate an upper limit of $<10^{-2}$ for the branching efficiency $\eta_{\mathrm{FM}}=\mathrm{k}_{\mathrm{FM}} / \mathrm{k}_{\mathrm{F}}$ from $\mathrm{F}$ to $\mathrm{M}$. With the apparent fluorescence quantum yield of $10^{-5}$, the detailed quantum yield $\eta_{M R}=k_{M R} /\left(k_{M R}+k_{M N R}\right)$ can be estimated to $>10^{-3}$, where $\mathrm{k}_{\mathrm{MR} / \mathrm{MNR}}$ are the radiative and non-radiative decay rates from $\mathrm{M}$. With the total decay rate of $\mathrm{F}, \mathrm{k}_{\mathrm{F}}=(1.3 \mathrm{ps})^{-1}$, we may state $\mathrm{k}_{\mathrm{FM}}>(130 \mathrm{~ns})^{-}$ 


\section{Conclusions}

In this Letter, we have reported new results on the photophysics of DTE, a diarylethene featuring a common methoxyphenyl substituent and a carboxylic anchoring group. The low fluorescence quantum yield of the c-form under steady state excitation would normally classify this isomer as non-fluorescent. Using highsensitivity methods and high temporal resolution, we were able to identify several emission channels which help to clarify the level structure of the molecule. The comparison with femtosecond transient absorption measurements revealed a formerly unnoticed metastable state which could in principle be related to the photoreaction. Also by comparison, we could associate an intermediate decay constant with ground state cooling. As it appears, c-form luminescence is more common in diarylethenes than previously thought. It may be worthwhile to inspect other diarylethene derivatives on a time-resolved basis in order to clarify the processes evolving during the photoreaction.

\section{Acknowledgments}

This work has been supported by the Deutsche Forschungsgemeinschaft (Sonderforschungsbereich 658). 


\section{References}

[1] R. B. Woodward, R. Hoffmann, Angew. Chemie Int. Ed. 8 (1969) 781-853.

[2] A. Staykov, K. Yoshizawa, J. Phys. Chem. C 113 (2009) 3826-3834.

[3] S. Shim, T. Joo, S. C. Bae, K. S. Kim, E. Kim, J. Phys. Chem. A 107 (2003) $8106-8110$.

[4] B. Heinz, S. Malkmus, S. Laimgruber, S. Dietrich, C. Schulz, K. RueckBraun, M. Braun, W. Zinth, P. Gilch, J. Am. Chem. Soc. 129 (2007) 85778584.

[5] Y. Ishibashi, M. Fujiwara, T. Umesato, H. Saito, S. Kobatake, M. Irie, H. Miyasaka, J. Phys. Chem. C 115 (2011) 4265-4272.

[6] S. Shim, I. Eom, T. Joo, E. Kim, K. S. Kim, J. Phys. Chem. A 111 (2007) $8910-8917$.

[7] K. Uchida, D. Guillaumont, E. Tsuchida, G. Mochizuki, M. Irie, A. Murakami, S. Nakamura, J. Mol. Struct. THEOCHEM 579 (2002) 115120.

[8] K. Yagi, M. Irie, Chem. Lett. 32 (2003) 848-849.

[9] G. Porter, C. Tredwell, Chem. Phys. Lett. 56 (1978) 278-282.

[10] T. Kawai, M.-S. Kim, T. Sasaki, M. Irie, Opt. Mat. 21 (2003) 275-278.

[11] M. Zastrow, S. Thyagarajan, S. Ahmed, P. Haase, S. Seedorff, D. Gelman, J. Wachtveitl, E. Galoppini, K. Rueck-Braun, Chem. Asian J. 5 (2010) 12021212.

[12] A.J. Myles, N.R. Branda, Macromolecules 36 (2003) 298-303.

[13] M. Irie, K. Sayo, J. Phys. Chem. 96 (1992) 7671-7674.

[14] E. Ippen, C. Shank, A. Bergman, Chem. Phys. Lett. 38 (1976) 611-614. 
[15] S. Pu, W. Liu, W. Miao, J. Phys. Org. Chem. 22 (2009) 954-963.

[16] S. Pu, G. Liu, G. Li, R. Wang, T. Yang, J. Mol. Struct. 833 (2007) 23-29.

[17] M. Irie, T. Lifka, K. Uchida, S. Kobatake, Y. Shindo, Chem. Commun. (1999) 747-750.

[18] J. Ern, A. Bens, H.-D. Martin, S. Mukamel, D. Schmid, S. Tretiak, E. Tsiper, C. Kryschi, Chem. Phys. 246 (1999) 115-125.

[19] K. Kuldova, K. Tsyganenko, A. Corval, H. Trommsdorff, A. Bens, C. Kryschi, Synth. Met. 115 (2000) 163-166.

[20] N. Tanaka, C. Okabe, K. Sakota, T. Fukaminato, T. Kawai, M. Irie, A. Goldberg, S. Nakamura, H. Sekiya, J. Mol. Struct. 616 (2002) 113-118.

[21] T. Fukaminato, T. Kawai, S. Kobatake, M. Irie, J. Phys. Chem. B 107 (2003) 8372-8377.

[22] T. Brust, S. Malkmus, S. Draxler, S. A. Ahmed, K. Rueck-Braun, W. Zinth, M. Braun, J. Photochem. Photobiol. A: Chem. 207 (2009) 209-216.

[23] A. de Bettencourt-Dias, A. Poloukhtine, J. Phys. Chem. B 110 (2006) 2563825645.

[24] M. Irie, O. Miyatake, K. Uchida, J. Am. Chem. Soc. 114 (1992) 8715-8716.

[25] C. Yun, J. You, J. Kim, J. Huh, E. Kim, J. Photochem. Photobiol. C: Photochem. Rev. 10 (2009) 111-129.

[26] A. Nakamura, T. Ohashi, K. Yamamoto, J. Ishihara, T. Aoki, J. Temmyo, H. Gotoh, Appl. Phys. Lett. 90 (2007) 093512-3.

[27] J. Ern, A. Bens, A. Bock, H.-D. Martin, C. Kryschi, J. Lumin. $76-77$ (1998) 90-94.

[28] C. Okabe, T. Nakabayashi, N. Nishi, T. Fukaminato, T. Kawai, M. Irie, H. Sekiya, J. Phys. Chem. A 107 (2003) 5384-5390. 


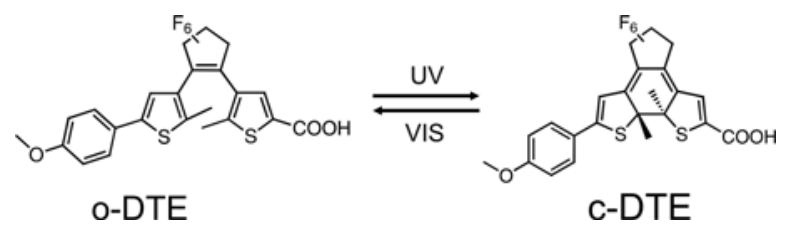

Scheme 1. Chemical structure of DTE in the open ring form (o-DTE) and the closed ring form (c-DTE). The photoinduced cyclization (cycloreversion) reaction is triggered by light in the UV (VIS) spectral range. 


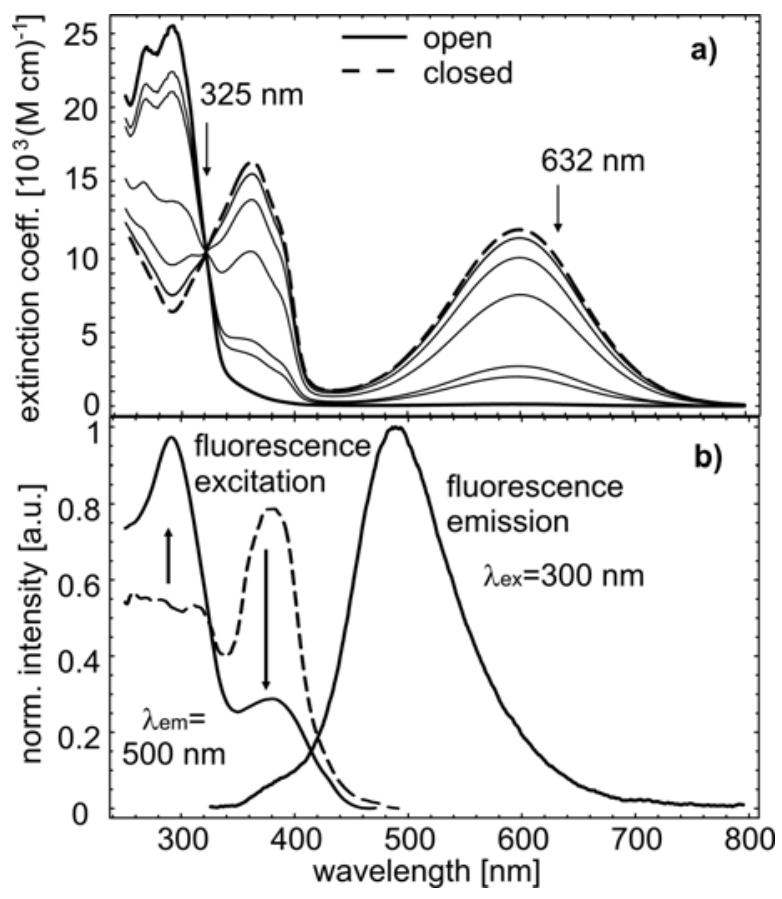

Figure 1. a): Steady state absorption spectra of DTE dissolved in chloroform (concentration $2 \times 10^{-6} \mathrm{M}$ ) in the o-form (bold, solid line) and in the c-form (bold broken line, PSS $>99 \%$ ). The weak lines indicate intermediate spectra recorded during photoconversion induced by excitation at 325 and $632 \mathrm{~nm}$. b): Blue-green fluorescence spectrum (solid line) of DTE excited at $300 \mathrm{~nm}$ and fluorescence excitation spectra for the c- (broken line) and the o-forms (solid line) monitored at $500 \mathrm{~nm}$. The concentration was $10^{-4} \mathrm{M}$. 


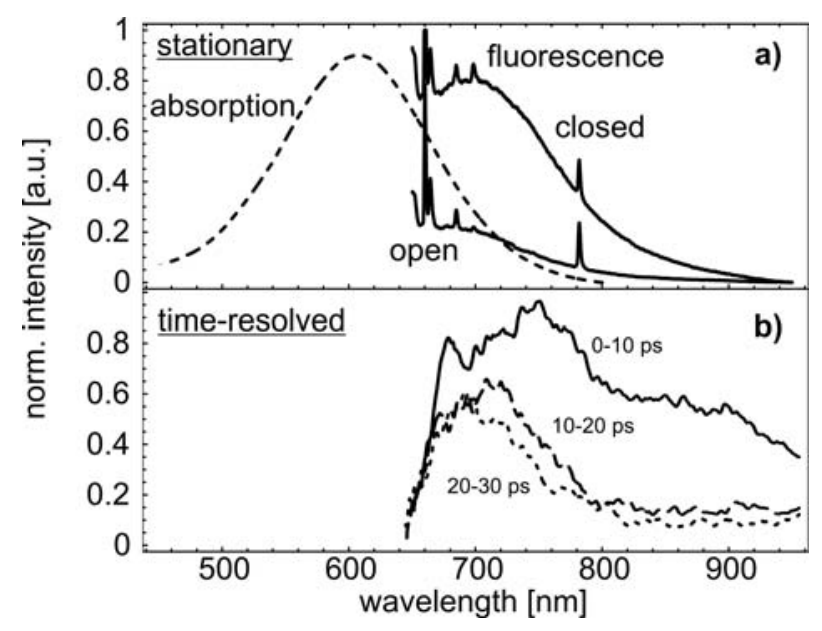

Figure 2.a): Steady state emission spectra (solid lines) after excitation at $632 \mathrm{~nm}$ of DTE (concentration $2 \times 10^{-4} \mathrm{M}$ ) in the c- and o- forms. The spikes are Raman lines from the solvent (chloroform). For comparison, the absorption spectrum (broken line) of c-DTE is displayed . b): Time resolved emission spectra of c-DTE (concentration $1.3 \times 10^{-4} \mathrm{M}$ ) at different temporal delays after excitation with a $100 \mathrm{fs}$ pulse centered at $628 \mathrm{~nm}$. Temporal integration was applied over 0-10 ps (solid), 10-20 ps (dashed) and 20-30 ps (dotted). The spectral shape remained unchanged for longer delays. 


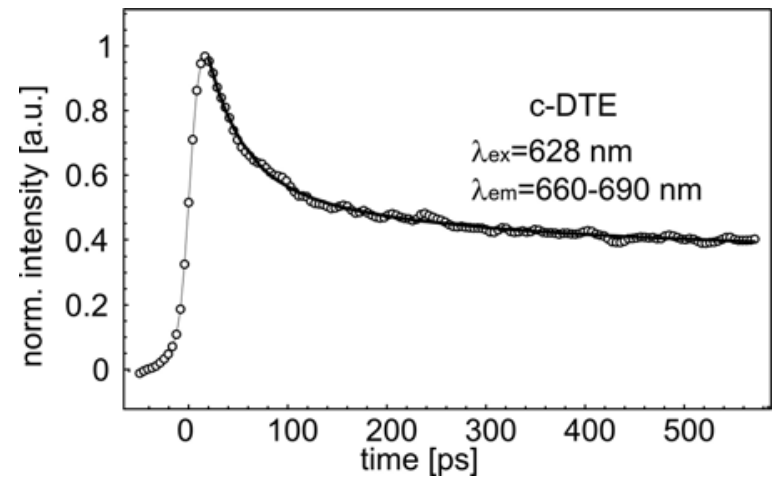

Figure 3. Time-resolved emission of c-DTE measured with excitation at $628 \mathrm{~nm}$. The emission intensity spectrally integrated between 660 and $690 \mathrm{~nm}$ is plotted as a function of time. 

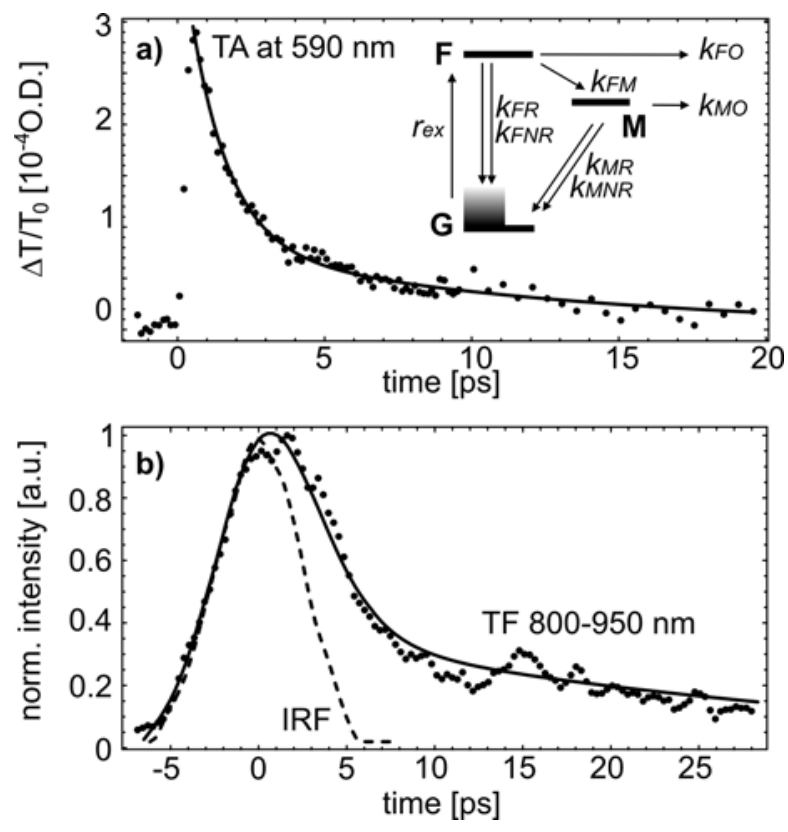

Figure 4. a): Time-resolved transmission change $\Delta \mathrm{T} / \mathrm{T}_{0}=\left(\mathrm{T}-\mathrm{T}_{0}\right) / \mathrm{T}_{0}$ of c-DTE (concentration $8 \times 10^{-4} \mathrm{M}$ ) measured in a femtosecond pump-probe experiment with pulses centered at $590 \mathrm{~nm}$ (symbols; $\mathrm{T}$ and $\mathrm{T}_{0}$ : sample transmission with and without excitation). The solid line is a biexponential fit with decay times of $1.3 \mathrm{ps}$ and $12 \mathrm{ps}$. Inset: Level scheme of c-DTE as deduced from the steady state and transient spectra (see text for details). b): Transient emission intensity of c-DTE after $628 \mathrm{~nm}$ excitation integrated over the spectral range from 800 to $950 \mathrm{~nm}$. The solid line is a biexponential decay with time constants of 2 ps and 36 ps convolved with the instrumental response (broken line, IRF). 


\section{Supplementary Information}

\section{Analytical Data for DTE}

m.p.: $108-110{ }^{\circ} \mathrm{C}, R_{\mathrm{f}}=0.6$ (ethylacetate $/ \mathrm{AcOH} 80: 1$ ),

${ }^{1} \mathrm{H}$ NMR $\left(400 \mathrm{MHz}, \mathrm{CDCl}_{3}\right): \delta=7.89$ (br. s, $\left.1 \mathrm{H}\right), 7.47-7.43(\mathrm{~m}, 2 \mathrm{H}), 7.11$ (br.

s, $1 \mathrm{H}), 6.94-6.90(\mathrm{~m}, 2 \mathrm{H}), 3.84(\mathrm{~s}, 3 \mathrm{H}), 2.01(\mathrm{~s}, 3 \mathrm{H}), 1.92(\mathrm{~s}, 3 \mathrm{H}) \mathrm{ppm}$;

${ }^{13} \mathrm{C}$ NMR $\left(100 \mathrm{MHz}, \mathrm{CDCl}_{3}\right.$ [The resonances of the carbon atoms of the fluorinated cyclopentene moiety of the compound could not be observed because of the low intensity and extensive coupling.]): $\delta=166.1,159.8$, $150.6,142.9,140.5,135.1,130.1,127.1,126.5,126.1,125.3,121.0,114.6$, 55.6, 15.2, $14.7 \mathrm{ppm}$.

IR (ATR): $\widetilde{v}=3002,2959,2934,2856,2840,1684,1610,1550,1515,1464$, $1441,1338,1273,1254,1192,1180,1139,1119,1044,1038,986,900,890$, $824 \mathrm{~cm}^{-1}$;

HRMS (ESI, negative mode) calcd for $\mathrm{C}_{23} \mathrm{H}_{16} \mathrm{~F}_{6} \mathrm{O}_{3} \mathrm{~S}_{2}\left[M^{+}-\mathrm{H}\right]$ : 517.0361; found: 517.0358 . 